\title{
Study of 0.3-0.8 THz flux generated by magnetized plasma column due to relaxation of high-current REB
}

\author{
$\underline{\text { A.V. Arzhannikov }}^{1,2}$, V.V. Annenkov ${ }^{1,2}$, A.V. Burdakov ${ }^{1,3}$, V.S. Burmasov ${ }^{1,2}$, I.A. Ivanov ${ }^{1,2}$, \\ A.A. Kasatov ${ }^{1,2}$, S.A. Kuznetsov ${ }^{1,2}$, M.A. Makarov ${ }^{1}$, K.I. Mekler ${ }^{1}$, S.V. Polosatkin ${ }^{1,2}$, \\ V.V. Postupaev ${ }^{1,2}$, A.F. Rovenskikh ${ }^{1}$, S. L. Sinitsky ${ }^{1,2}$, V.F. Sklyarov ${ }^{1,2}$, V.D. Stepanov ${ }^{1,2}$, \\ I.V. Timofeev ${ }^{1,2}$, and E.P. Volchok ${ }^{2}$ \\ ${ }^{1}$ Institute of Nuclear Physics, SB RAS, Novosibirsk, Russia, arzhan1@ngs.ru \\ ${ }^{2}$ Novosibirsk State University, Novosibirsk, Russia \\ ${ }^{3}$ Novosibirsk State Technical University, Novosibirsk, Russia
}

\begin{abstract}
Studies on submm-wave emission from magnetized plasma due to relaxation of a high current relativistic electron beam (REB) are considerably important since they allow generating the high-power radiation with a prompt tuning frequency $[1,2]$. The experimental results were obtained at GOL-PET device (see fig. 1). We study the relaxation of the high-power REB $(0.8 \mathrm{MeV} / 10 \mathrm{kA} /$ $10 \mu \mathrm{s})$ in a plasma column of 2 meters length with the density $\mathrm{n}_{\mathrm{p}}=(0.5-2) 10^{15} \mathrm{~cm}^{-3}$ confined by the multiplemirror magnetic field with the mean value $B_{\text {mean }}=4.2 \mathrm{~T}$ and the corrugation factor 1.5. The aim of the experiments is to find the conditions for the efficient conversion of plasma waves into electromagnetic ones. The recent experiments have been focused on the study of the properties of $0.2-0.8 \mathrm{THz}$ radiation propagating along the axis of the plasma column.

The temporal dynamics of the radiation spectra was measured along the axis by an 8-channel polychromator with semiconductor diodes for the frequency interval from 0.1 up to $0.5 \mathrm{THz}$ (see [2]) and by an additional 2channel cryogen detection system and single semiconductor diodes covered by narrow band-pass quasi-optical filters for the $0.5-0.9 \mathrm{THz}$ frequency range. During the spectral measurements, the plasma density was measured in eight dots over the diameter of the plasma column via the Thomson scattering system with a $50 \mathrm{~ns}$ laser pulse.
\end{abstract}

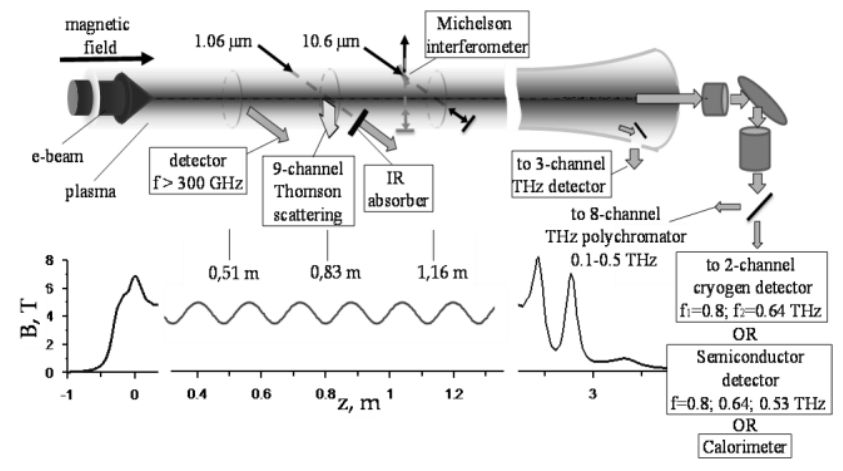

Fig. 1. The layout of the GOL-PET experiments

As an example of the results of such measurements by the Thomson scattering system, the density distribution over the plasma column diameter is presented in fig. 2 for the shot $\mathrm{t} 1913$.

The temporal evolution of beam and plasma parameters and the signals of the emission for the frequencies $0.26 \mathrm{THz}$ and $0.64 \mathrm{THz}$ are presented in fig. 3 for the same shot.
Measurements of the emission in the longitudinal direction have shown that the spectral power density of this radiation is concentrated in two clear distinct frequency regions as it was demonstrated in the measurements of the emission in transverse direction (see [3]). For the plasma density of $110^{15} \mathrm{~cm}^{-3}$, the first region is located in the frequency interval $\mathrm{f}_{1}=0.25-0.35 \mathrm{THz}$, the second one is in the interval $\mathrm{f}_{2}=0.6-0.8 \mathrm{THz}$. As one can see in fig. 4 presenting the spectrum of the emission for the shot $\mathrm{t} 1913$, one of the peaks of the spectral power density is located at the frequency $\mathrm{f}_{1}=0.2 \mathrm{THz}$ and the second one at $\mathrm{f}_{1}=0.64 \mathrm{THz}$.

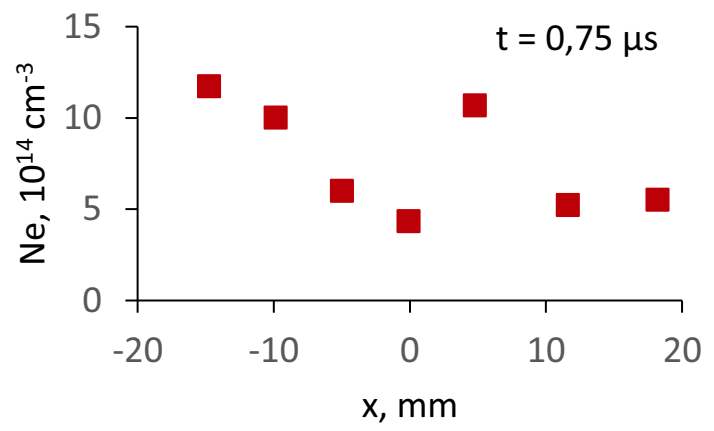

Fig. 2. Plasma density distribution over the column diameter measured in the shot 1913 for the time of the maximal $\mathrm{THz}$ emission

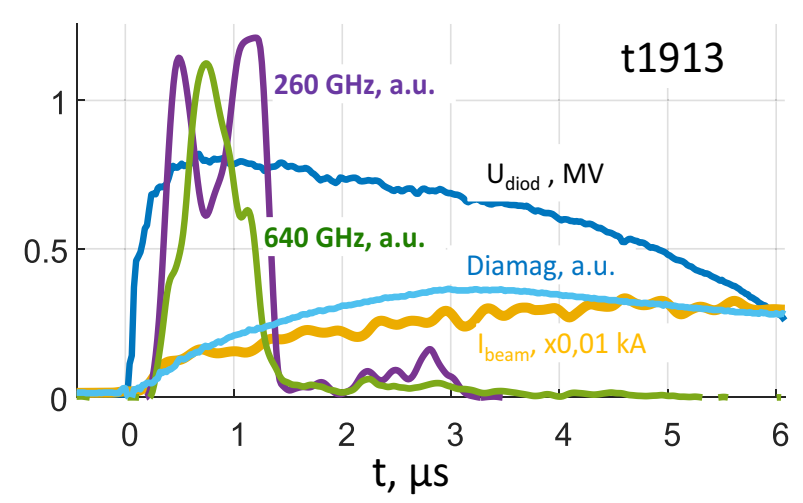

Fig. 3. Temporal evolution of the main beam and plasma parameters and the signals of the $\mathrm{THz}$ emission

We have compared the experimental results with the theoretical consideration of the transformation of the plasma oscillations into the electromagnetic radiation for the conditions of our experiments [4-5]. The locations of the frequency ranges with the high level of the spectral power density are associated with the frequency of the 
upper hybrid mode of plasma oscillations and the doubled value of this frequency.

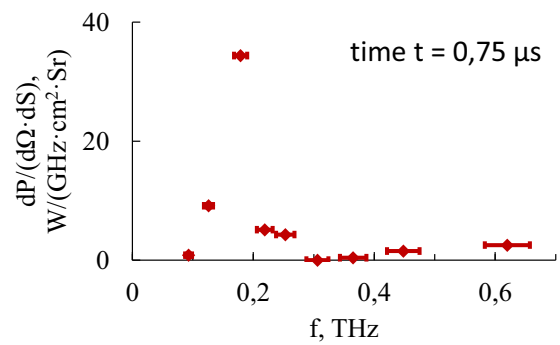

Fig. 4. Spectral density of the radiation measured in shot T1913 at the moment of maximum $\mathrm{THz}$ emission

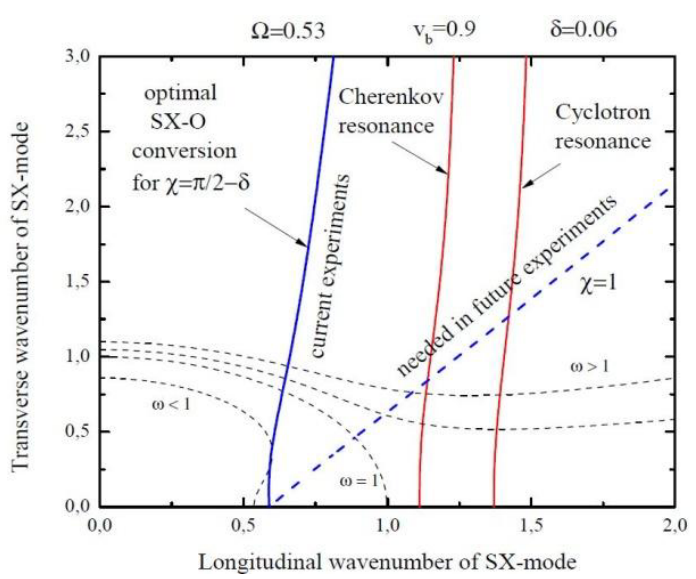

Fig. 5. Lines of the wave numbers of the upper hybrid oscillations in the areas of the resonance with the relativistic electron beam (red lines) and of their effective transformation into the electromagnetic radiation (blue lines)

Taking into account our experimental and theoretical studies on the REB-plasma interaction, we can conclude that the observed emission is explained by the direct transmission of the upper hybrid branch of plasma oscillations to the electromagnetic radiation [4] and merging of two upper hybrid oscillations of the high level plasma turbulence [5]. In current experiments, the line optimal SX-O mode conversion in the wavenumber space is shifted from the area of the intense pumping of the upper hybrid wave by the REB (see fig. 5). The reason for that is almost transverse orientation of magnetic field lines (even in the corrugated regime) to the predominantly radial density gradient. In future experiments, we plan to realize the regime in which the density gradient is directed at an acute angle to the magnetic field. The regime of $\chi=1$ needed to convert the most unstable beam-driven modes is shown in fig. 5 by the blue dashed line.

So, the REB-plasma interaction is the sufficient method to generate sub millimeter radiation for frequency range up to $1 \mathrm{THz}$. This method is based on the intense pumping of the upper hybrid waves by the REB and then the transformation of these waves to the emission through the SX-O mode conversion and the merging of two upper hybrid waves.

The work was supported by Russian Science Foundation in the frame of Project No. 14-12-00610 for experimental and theoretical studies of the spectral composition of the emission and in the frame of Project No. 14-5000080 for the reconstruction of the GOL-PET device.

\section{References}

1. M.K.A. Thumm, A.V. Arzhannikov, V.T. Astrelin et al., Generation of High-Power Sub-THz Waves in Magnetized Turbulent Electron Beam Plasmas // Journal of Infrared, Millimeter and Terahertz Waves. 2014, Vol. 35, Iss. 1, pp. 81-90, DOI 10.1007/s10762-013-9969-3.

2. A.V. Arzhannikov, A.V. Burdakov, V.S. Burmasov, et al., Dynamics and Spectral Composition of Subterahertz Emission From Plasma Column Due to Two-Stream Instability of Strong Relativistic Electron Beam // IEEE Transactions on Terahertz Science and Technology, 2016, Vol. 6, No. 2, P.245.

3. A.V. Arzhannikov, V.V. Annenkov, A.V. Burdakov, et al., Beam-plasma system as a source of powerful submillimeter and terahertz radiation (experimental and theoretical studies) // AIP Conf. Proc., 1771, 070004 (2016); http://dx.doi.org/10.1063/ 1.4964228.

4. I.V. Timofeev, V.V. Annenkov, A.V. Arzhannikov, Regimes of enhanced electromagnetic emission in beam-plasma interactions // Physics of Plasmas, 2015, 22, p.113109, http://dx.doi.org/10.1063/1.4935890.

5. A.V. Arzhannikov and I. V. Timofeev. Generation of powerful terahertz emission in a beam-driven strong plasma turbulence // Plasma Phys. Controlled Fusion, 2012, 54, 105004. http://dx.doi.org/10.1088/0741-3335/54/10/105004. 